\title{
Possible association of ultra-high-energy cosmic-ray events with strong gamma-ray bursts
}

\author{
Mordehai Milgrom and Vladimir Usov \\ Department of Condensed-Matter Physics, Weizmann Institute of Science 76100 Rehovot, Israel
}

\begin{abstract}
We point out that each of the error boxes of the two highest-energy cosmic-ray shower events known, overlaps with that of a strong gamma-ray burst (GRB). The GRBs precede the cosmic rays by 5.5 , and 11 months respectively. In one case the strongest known cosmic ray is paired with the strongest gamma-ray burst in the BATSE catalogue. The probability of this to have occurred by chance seems rather small. Without building on post-factum statistics, we think the above is remarkable enough to suggest that the cosmic ray and gamma-ray burst were produced by the same outburst. A time delay (and a small positional disparity) is expected, since the trajectory of a charged cosmic-ray particle is wriggled by intervening magnetic fields. We estimate that the Galaxy's field alone may produce a delay of the order observed. With similar energies going into gamma rays and cosmic rays, we expect only a few tens percent of the strongest GRBs to be followed by a detection of an ultra-high-energy cosmic ray in existing experiments. We discuss some of the implications that follow if such an association is confirmed. For example, the upper limit on the distance of the cosmic-ray source, combined with a much-better-determined position to the gamma-ray burst source, narrows greatly the volume in which to look for an optical counterpart. The need to produce ultra-high-energy cosmic rays in the GRB source imposes additional constraints on the physical conditions in it. There is also useful information in the time delay regarding, e.g., intergalactic magnetic fields.
\end{abstract}

Subject headings: Gamma-ray bursts, ultra-high-energy cosmic rays, cosmic rays

\section{Introduction}

The nature of the events that engender cosmic gamma-ray bursts (GRB) has not been established yet; even their rough whereabout on the cosmic map is still a moot issue (for a review, see Hartmann 1994). While various constraints have been devised regarding physical characteristics of the underlying process, no definite picture has emerged. As is the rule in astronomy, important further clues would be come by if the GRB source could be identified as a 
generator of a different type of signals (e.g. of electromagnetic radiation in the optical region). A similar situation exists regarding the (very few) ultra-high-energy cosmic-ray (UHECR) events of energy higher than $10^{20} \mathrm{eV}$. The situation concerning optical identification of the sources of UHECRs, some constraints on their origin, and physical conditions in their sources have been recently summarized by Sigl, Schramm \& Bhattacharjee (1994), and by Halzen et al. (1995).

We have set to check whether a connection can be made between these two types of phenomena. Expecting a time delay, we looked for GRBs that precede UHECR events by at most a year or two, and that come from the very near vicinity. If the association we conjecture here is confirmed the constraints that apply to each phenomenon separately can be cast on the other.

\section{Cosmic-ray-GRB association}

In looking for associations with GRBs we have concentrated on only the most energetic UHECRs, for the following reasons. The higher the energy of a charged cosmic ray $(\mathrm{CR})$, the less its trajectory is affected by intervening magnetic fields, and the more practicable it is to associate it with a GRB: First, trajectory wriggling causes the CR to appear from a false position. Sigl, Schramm \& Bhattacharjee (1994) estimate the disparity in angle to be at most a few degrees for the most energetic UHECRs $\left(E \sim 3 \times 10^{20} \mathrm{eV}\right.$ ) (see also $\S 3$ ), but the expected deflection angle scales in inverse proportion to the energy. Second, since we are interested in correlations with brief, temporal events we must also reckon with the fact that trajectory wriggling produces a time delay in the arrival time of the CR. We discuss this in $\S 3$, but note here only that for the very-highest-energy CRs the expected time delay is already of the order of a few months, at the minimum, and the delay scales as the inverse square of the energy (and is very uncertain). For lower-energy CRs there may be too long a time stretch in which to look for candidate GRB's with any degree of confidence, without refined statistical tools. Third, because the energy of UHECRs degrades quickly with propagation through the intergalactic medium (e.g. Puget, Stecker, \& Bredekamp 1976, Hill \& Schramm 1985, Sigl, Schramm, \& Bhattacharjee 1994) the highest-energy ones are probable to come from nearer by. A GRB that comes from the same source is then expected to be stronger (of higher flux), thus rarer among the GRB's, and, in turn, more confidentially associated with the UHECR. In general, such more energetic events are also better pin-pointed on the sky in both the CR and gamma-ray regime because of better statistics. (For strong GRBs there is added bonus in that they have a better chance of being detected by the higher-energy instruments such as EGRET, with an even better angular accuracy.)

There are very few UHECRs reported in the literature with energies above $10^{20} \mathrm{eV}$. In the pre-1991 era-for which we have not had available systematic GRB data to look in-there is the event detected by the Yrkutsk experiment, in May 1989 with energy of $(1.2 \pm 0.4) \times 10^{20} \mathrm{eV}($ Efimov et al. 1991), and 4 events from the Haverah-Park experiment described in (Brooke et al. 1985) 
- which reference has not been available to us (their existence is reported in Lawrence, Reid, \& Watson 1991). We are then left with only two UHECR events observed from 1991 onward: The most energetic event ever detected was seen by the Fly's Eye experiment on October 151991 , with $E=\left(3.0_{-0.54}^{+0.36}\right) \times 10^{20} \mathrm{eV}$ (Bird et al. 1993, Bird et al. 1994). The position and error box $\left(\alpha=85.2^{\circ} \pm 1^{\circ}, \delta=48^{\circ} \pm 10^{\circ}\right)$ are shown in Fig. 2 of Sigl, Schramm, \& Bhattacharjee 1994 in galactic coordinates. The second most energetic UHECR was detected by the AGASA experiment on December 31993 , with $E=(1.7-2.6) \times 10^{20} \mathrm{eV}$, and position: $\ell^{I I}=131^{\circ}, b^{I I}=-41^{\circ}$, with an error circle $(1-\sigma)$ of $1^{\circ}$ Yoshida et al. 1995).

We searched the BATSE catalogues (Fishman et al. 1994, Meegan et al. 1994) for GRBs observed before each of the two UHECR events, and within a few degrees of its reported position.

For the Fly's Eye event (hereafter FEE) we found within the error box a single BATSE GRB (910503) that was observed about 5.5 months earlier (the BATSE record starts only about a fortnight earlier yet). This event happens to be the highest-total-fluence event in the BATSE catalogue, and in all respects it is among the very few brightest GRBs observed to date. It is the 1991 May 3 event that was detected also by COMPTEL (Winkler et al.1992a), by EGRET (Schneid et al.1992, Hurley et al. 1994), and by Ulysses, and PVO (Fishman et al. 1994, Varendorff et al. 1992). A maximum photon energy of $10 \mathrm{GeV}$ is reported (Hurley et al. 1994). The best single-instrument position comes from EGRET (Schneid et al.1992): $\ell^{I I}=171.9^{\circ} \pm 1.3^{\circ}$, $b^{I I}=5.3^{\circ} \pm 1.1^{\circ}$. That of COMPTEL is (Winkler et al.1992a): $\ell^{I I}=171.8^{\circ}, b^{I I}=6.4^{\circ}$, with an error radius of about $3^{\circ}$, all errors are for $99 \%$ confidence level. Time delay measurements with PVO and/or Ulysses can give a much better angular definition. Indeed for GRB 910503 Varendorff et al. 1992 cite a combined COMPTEL-Ulysses error box of only $1^{\circ} \times 1^{\prime}$, which, we find, overlaps with the UHECR error box. The different error regions are depicted in Fig. 6 of Winkler et al. 1992b. The error box of the FEE covers a fraction of about $10^{-3}$ of the sky, and the chance probability to find in that box at least one of the $~ 85$ BATSE events that preceded the cosmic ray is small, to say nothing of the probability to find a rare event such as GRB 910503.

As to the AGASA event, in its near vicinity (a few degrees) we found no GRB among the 260 in the first BATSE catalogue (recording starts 21 months before the CR event). In the second BATSE catalogue (Meegan et al. 1994) we found three GRBs: 1. GRB 920615B (June 15 1992) at $\ell^{I I}=129.2^{\circ}, b^{I I}=-39.62^{\circ}$. It has no quoted fluxes, but the rather large quoted photon-counting positional error of $4.15^{\circ}$ indicates that it was not energetic. 2. GRB 920617C (June 17 1992-perhaps related to the previous) from $\ell^{I I}=131.18^{\circ}, b^{I I}=-41.25^{\circ}$, is also energetically inconspicuous (photon counting positional error of $5.59^{\circ}$ ). 3. GRB 921230 (December 30 1992-11 months before the AGASA event), with a position $\ell^{I I}=132.91^{\circ}, b^{I I}=-42.87^{\circ}$ (photon-counting positional error of $0.4^{\circ}$ ) is about $3^{\circ}$ away from the center of the CR error box. While the GRB statistical position error in this case is small, BATSE is said to have a systematic uncertainty of $4^{\circ}$ (Fishman et al. 1994, Meegan et al. 1994). Unlike the other case we do not have here the benefit of a more accurate EGRET or COMPTEL position, so the association remains even more tentative. Energetically, GRB 921230 is not in the same league as GRB 910503 (it has 
a total BATSE fluence about seven times smaller) but is definitely among the top ten percent in fluence.

Pitfalls of post-factum statistics abound in the present case. We believe, however, that the facts as described above cogently bring to mind the possibility that the UHECR event, and the GRB are engendered by the same cataclysmic event. At any rate, the exact statistical significance of the association need not be established at this point. More correlations such as those above are expected for future pairs. In fact, some strong GRBs that have been observed recently (e.g. Hurley et al. 1994 may be good candidate precursors for future UHECR events.

\section{Propagation effects}

UHECRs are almost certainly extragalactic. Proton seems to be favoured as the primary particle that triggers the event (Sigl, Schramm, \& Bhattacharjee 1994), but a neutrino cannot be excluded as an atypical primary (Sigl, Schramm, \& Bhattacharjee 1994, Halzen et al. 1995). We assume the former in what follows presently; neutrinos will be discussed at the end of this section.

Two effects on an UHECR proton traveling though the galactic and extragalactic medium have been extensively discussed in the literature, to wit energy loss, and angular deflection (for recent accounts see e.g. the above): A proton with energy above $10^{20} \mathrm{eV}$ loses energy effectively mainly by pion production on diffuse-background, low-energy photons. For example, for arrival energy of $1.7 \times 10^{20} \mathrm{eV}$, the initial energy has to be $\sim 10^{21} \mathrm{eV}$ for an origin distance of $D \simeq 100 \mathrm{Mpc}$, and $\sim 10^{25} \mathrm{eV}$ for $D \simeq 200 \mathrm{Mpc}$. Such energies are very taxing for any conceivable acceleration mechanism. It is believed, then, that the distance to the sources of the FEE and AGASA events cannot be more than $\sim(100-200)$ Mpc.

The particles produced by the above processes, especially ultra-high-energy photons, may also be detected on earth in positional or temporal correlation with GRBs. We intend to discuss these separately.

The second effect is the change in the apparent direction of the particle due to Larmour curving of the trajectory by intervening magnetic fields. For a homogeneous field, and a constant CR energy, the disparity angle is

$$
\alpha \simeq \frac{D}{2 r_{L}} \simeq 0.8\left(\frac{D}{3 \mathrm{kpc}}\right)\left(\frac{E}{10^{20} \mathrm{eV}}\right)^{-1}\left(\frac{B_{\perp}}{10^{-6} \mathrm{G}}\right) \mathrm{deg},
$$

where $r_{L}=E / e B_{\perp}$ is the gyroradius for a proton of energy $E$, and $B_{\perp}$ is component of the magnetic field perpendicular to the line of sight. In a tangled field the process is one of random walk in the angle. Within each cell of roughly homogeneous field (of mean size $d_{c}$-the reversal length) the spread in angles increases by an amount given by eq.(1) with $D=d_{c}$. The random-walk 
step increases as $E$ decreases along the trajectory, and the effect has to be integrated along the line of sight.

In the context of the UHECR-GRB association we note that trajectory wriggling also produces a time delay of the CR particle. Unlike the angle deviation, which random walks, the time delay only increases with length of travel. For a homogeneous field, constant energy, and small departure from the line of sight, the time delay is

$$
\Delta t \simeq \frac{1}{24} \frac{D^{3}}{c r_{L}^{2}} \simeq 0.3\left(\frac{D}{3 \mathrm{kpc}}\right)^{3}\left(\frac{E}{10^{20} \mathrm{eV}}\right)^{-2}\left(\frac{B_{\perp}}{10^{-6} \mathrm{G}}\right)^{2} \mathrm{yr} .
$$

(Geometrical factors of order of a few may enter in other geometries). In this case $\Delta t$ is related to the disparity angle $\alpha$ by

$$
\Delta t=\frac{1}{6} \frac{D \alpha^{2}}{c}
$$

When the magnetic field is tangled, a simple estimate of the effect can be obtained by picturing the line of sight to be divided into segments of mean length $d_{c}$ over which the magnetic field is constant. The minimum time delay will be produced when the particle returns to the line of sight within each cell (the particle could wander off the line of sight and return only upon arrival, in which case the time delay could be much larger). Then $\Delta t_{\text {min }} \sim \int d \delta t$, where

$$
d \delta t \simeq 0.3\left(\frac{d D}{3 \mathrm{kpc}}\right)\left(\frac{E}{10^{20} \mathrm{eV}}\right)^{-2}\left(\frac{B_{\perp}}{10^{-6} \mathrm{G}}\right)^{2}\left(\frac{d_{c}}{3 \mathrm{kpc}}\right)^{2} \mathrm{yr}
$$

is the delay accumulated over path length $d D$; we assume that $d_{c}$ is small compared with the length over which $E$ varies appreciably.

In principle, propagation effects as discussed above may arise in the galaxy hosting the source, in our galaxy, and in the IGM. On the scale of a galaxy there is hardly a loss of energy, so constant $E$ may be assumed in the above estimates. Magnetic field effects in the host galaxy contribute negligibly to the angle disparity (in the present context).

Consider first the contribution of the Galaxy alone. The FEE particle comes roughly from the galactic anti-centre. From Fig. 1a of Vallee (1991), which shows a magnetic map of the relevant region, and from his Table 1, we see that it is appropriate to take, approximately, $B_{\perp} \sim(2-3) \mu \mathrm{G}$ and $D$ and $d_{c}$ of a few kiloparsecs. With the energy of the FEE particle eqs. (2) and (4) give a time delay of a few months consistent with association the FEE with GRB 910503. The AGASA CR comes from galactic latitude of $\sim 40^{\circ}$, so its trajectory cuts only about $2 \mathrm{kpc}$ through the galactic-disk field-which is said to have a scale hight of $\sim 1 \mathrm{kpc}$ (Kronberg 1994)-but its energy is smaller, and we expect a similar galactic time delay (and halo fields may also contribute). The corresponding spread in angles is about $1^{\circ}$.

The extragalactic contribution is much more uncertain. In this connection note that the CR particle is expected to alternate in identity between a proton and a neutron due to the pion-producing interactions, so it is only during about half of its journey that it is subject to 
magnetic field effects. (The chances for identity change in the galaxy are small, and we can ignore the effect in the above estimate.) Second, because the incremental time delay decreases fast with increasing energy, only the end part of the intergalactic journey contributes appreciably to it (assuming similar magnetic fields along the way). We see from Fig. 1 of Sigl, Schramm \& Bhattacharjee (1994), which shows the decrease of energy with travel length, that only about the last $(25-50) \mathrm{Mpc}$ need be taken into account; this should be halved due to the proton-neutron alternations.

Very little is known about the intergalactic field at large. Kronberg (1994) cites an upper limit of $10^{-9} \mathrm{G}$ on $B$ and of $1 \mathrm{Mpc}$ on $d_{c}$. Assuming these values, and an effective propagation distance of $25 \mathrm{Mpc}$, with an energy of $3 \times 10^{20} \mathrm{eV}$, we obtain from eq.(四) a minimum time delay of about 25 years. Thus, confirmation of the association, with a time delay of order of a year or less, will cast a useful constraint on $B d_{c}$ for the intergalactic magnetic field. Interestingly, in this context, Plaga (1995) has recently proposed to probe intergalactic magnetic fields in the range $\left(10^{-12}-10^{-24}\right) \mathrm{G}$ using the (rather shorter) time delay of high-energy gamma-rays. His analysis may be relevant to ultra-high-energy photons that are produced by the UHECRs on their way, e.g. from $\pi^{0}$ decay.

If the CR arrives to the galaxy as a neutron, only propagation effects in the IGM and host galaxy contribute. The primary CR may, after all, be a neutrino, produced by a proton in the host galaxy. As it propagates subsequently without disturbance, it is expected to arrive without energy loss, or angular disparity. The only contribution to a time delay may come from a host galaxy-the neutrino being produced by an accelerated proton, which is subject to the above magnetic-field effects in te host galaxy. In this case, one is freed from the energy-loss constraint on the distance. Sigl, Schramm \& Bhattacharjee (1994) argue that a neutrino primary can only be an atypical instance, and that for every neutrino event there must be many proton events with similar arrival energy.

\section{Discussion}

If the GRB-UHECR association is confirmed by future correlations, further insight into the nature of the underlying mechanism can be gained by combining the constraints we deduce, separately, for the two phenomena. For instance, the position of a strong GRB may be much better defined than that of an UHECR, and, furthermore, the GRB's position can be trusted as it is not affected by magnetic fields. In the case of FEE-GRB 910503 the combined $1^{\circ} \times 1^{\prime}$ error box of the latter is some 2500 times smaller in area than that of the former. With additional satellites, GRB error boxes may be reduced even further by an order of magnitude or two (as has been the case in the seventies). If it is also confirmed that UHECRs are protons, the energy-loss argument greatly constrain the distance of the source. Combined, the two constraints reduce greatly the volume in which to look for counterparts. 
If the GRB event is also to produce UHECR s, the presence of very strong magnetic fields will be implied. What little we know about the physical conditions existing in gamma-ray bursters during an outburst makes them highly conducive to the acceleration of UHECR. For example, by one class of models, GRBs are produced in differentially rotating disks that are formed by a merger of a neutron-star binary (Paczyński 1986; Mochkovitch et al. 1993; Piran 1994), or by neutron stars that have arisen from accretion-induced collapse (Usov 1992). The magnetic field, $B_{\mathrm{S}}$, at the surface of these objects may be as high as $\sim 10^{16}-10^{17} \mathrm{G}$ Narayan, Paczyński \& Piran 1992; Usov 1992; Thompson \& Duncan 1993). The angular velocity of these objects is $\Omega \sim 10^{4}$ $\mathrm{s}^{-1}$. The potential difference between the surface of such an object and infinity is (e.g., Ruderman \& Sutherland 1975

$$
\Delta \varphi_{\max }=\frac{\Omega^{2} B_{\mathrm{S}} R^{3}}{2 c^{2}} \simeq 1.7 \times 10^{23}\left(\frac{\Omega}{10^{4} \mathrm{~s}^{-1}}\right)^{2}\left(\frac{B_{\mathrm{S}}}{10^{16} \mathrm{G}}\right)\left(\frac{R}{10^{6} \mathrm{~cm}}\right)^{3} \mathrm{~V}
$$

where $R \simeq(1-2) \times 10^{6} \mathrm{~cm}$ is the radius of the object. Charged particles that flow away from the surface may be accelerated, in principle, up to the energy $E_{\max } \simeq e \Delta \varphi_{\max }$, which is more than is needed to produce the observed UHECRs, at a distance of up to $\sim 100 \mathrm{Mpc}$.

Alternatively, particles may be accelerated by relativistic shocks that may be formed in an unsteady relativistic wind (Rees \& Mészáros 1994). The maximum energy that may be achieved by protons in the process is (Sigl, Schramm, \& Bhattacharjee 1994 and references therein)

$$
E_{\max } \simeq 10^{17}\left(\frac{B r_{\mathrm{sh}}}{0.001 \mathrm{pc} \mathrm{G}}\right) \mathrm{eV},
$$

where $r_{\mathrm{sh}}$ is the characteristic size of the shock. In our case $r_{\mathrm{sh}}$ is about the distance from the compact object to the shock. In the wind, the value of $B r_{\mathrm{sh}}$ does not depend on the distance from the compact object, beyond the light cylinder, and is $\sim B_{\mathrm{S}}(\Omega R / c)^{3}(c / \Omega)=\Omega^{2} B_{\mathrm{S}} R^{3} / c^{2}$. We can thus see that $e \Delta \varphi_{\max }$ with $\Delta \varphi_{\max }$ from equation(5) coincides with $E_{\max }$ given by equation (6) within a factor of 2 or so.

If, as has been suggested, the intrinsic luminosity of GRBs is rather uniform, and thus fluence is strongly indicative of distance; and if, further, the UHECR luminosity is a given fraction of the gamma luminosity, then we expect the probability to detect a UHECR in association with a GRB to decrease steeply with decreasing GRB fluence. What is the total energy flux in UHECRs per one outburst? Given that only one particle was observed for each of the two GRBs we cannot, directly, estimate this quantity without knowing the probability to detect a UHECR per GRB. We deduce that this probability, for detectors of the size operating today, is rather smaller than unity, even for those GRBs as energetic as GRB 910503: If one UHECR per GRB was typical say for AGASA, with its area of about $100 \mathrm{~km}^{2}$ (Yoshida et al. 1995), the AGASA event would have indicated a total UHECR fluence of $\sim 3 \times 10^{-4} \mathrm{erg} \mathrm{cm}^{-2}$, at earth, compared with the total BATSE fluence of GRB 921230 of $\sim 4 \times 10^{-5} \mathrm{erg} \mathrm{cm}^{-2}$. In addition, UHECR energy degrades in travel, so this would have given a high ratio (perhaps as high as 100) of UHECR/GRB energy at the source. This high ratio can be avoided if the probability for AGASA to detect an UHECR for 
a GRB as energetic as GRB 921230 is at most a few percent; this would correspond to an energy ratio of order unity-not straining further the energetics of the GRB source. More pertinently, this is in keeping with the fact that a few tens of GRBs as strong as GRB 921230 are observed per year, with only one UHECR by AGASA over a few years. GRB 910503 is about seven times more energetic, and about a few tens times rarer, so we expect on such burst in a few to be detected in UHECR. In both cases, detection statistics is consistent with roughly similar energies going into UHECRs, and gamma-rays.

A remarkable gamma-ray burst, GRB 940217, was observed on Feb 17 1994. The total fluence above $20 \mathrm{keV}$ of $\simeq 6 \times 10^{-4} \mathrm{erg} \mathrm{cm}^{-2}$ is twice higher than that of GRB 910503. A photon with the energy of $18 \mathrm{GeV}$ was detected one-and-a-half hour after the BATSE burst trigger from the region of GRB 940217 (Hurley et al. 1994). This is the highest energy of photons that was ever observed in a burst. The direction is known quite well: a combined COMPTEL-EGRET-Ulysses error box of only $\sim 0.5^{\circ} \times 0.1^{\circ}$. This burst is very promising as a source of UHECRs. 


\section{REFERENCES}

Bird, D.J., et al. 1993, Phys. Rev. Lett., 71, 3401

Bird, D.J., et al. 1994, ApJ, 424, 491

Brooke, G., et al. 1985, Proc. 19th Intl. Cosmic Ray Conf. (La Jolla) 2, 150

Efimov, N.N., et al. 1991, in Astrophysical Aspects of the Most Energetic Cosmic Rays, eds. M. Nagano \& F. Takahara, World Scientific, Singapore, p. 20

Fishman, G.J., et al. 1994, ApJ Supp., 92, 229

Halzen, F., Vázquez, R.A., Stanev, T., \& Vankov, H.P. 1995, Astropart. Phys., 3, 151

Hartmann, D. 1994, in The Gamma-Ray Sky with Compton GRO and SIGMA, eds. M.Signore, P. Salati, \& G. Vedrenne, in press

Hill, C.T., \&, Schramm, D.N. 1985 Phys. Rev., D31, 564

Hurley, K., et al. 1994, Nature, 372, 652

Kronberg, P.P. 1994, Rep. Prog. Phys., 57, 325

Lawrence, M.A., Reid, R.J.O., \& Watson, A.A. 1991, J. Phys., G17, 773

Meegan, C.A., et al. 1994, BATSE 2B Gamma-Ray Burst Electronic Catalogue, available from gronews at grossc.gsfc.nasa.gov

Mochkovitch, R., Hernanz, M., Isern, J., \& Martin, X. 1993, Nature, 361, 236

Narayan, R., Paczyński, B., \& Piran, T. 1992, ApJ, 395, L83

Paczyński, B. 1986, ApJ, 308, L43

Piran, T. 1994, in Gamma-Ray Bursts, Second Workshop, eds. G.J. Fishman, J.J. Brainerd, \& K. Hurley (New York: AIP), p.543

Plaga, R. 1995, Nature, 374, 430

Puget, J.L., Stecker, F.W., \& Bredekamp, J.H. 1976, ApJ, 205, 638

Rees, M.J., \& Mészáros, P. 1994, ApJ, 430, L93

Ruderman, M.A. \& Sutherland, P.G. 1975, ApJ, 196, 51

Schneid, E.J., et al. 1992, A \& A Lett., 255, L13

Sigl, G., Schramm, D.N., \& Bhattacharjee, P. 1994, Astropart. Phys., 2, 401 
Thompson, C., \& Duncan, R.C. 1993, ApJ, 408, 194

Usov, V.V. 1992, Nature, 357, 472

Vallee, J.P. 1991, ApJ, 366, 450

Varendorff, M.G., et al. 1992, Proc. AIP Conf. 265: Gamma-Ray Bursts Huntsville. W.S. Paciesas \& G.J. Fishman Eds., p.77

Winkler, C., et al. 1992a, A \& A Lett., 255, L9

Winkler, C. et al. 1992b, Proc. AIP Conf. 265: Gamma-Ray Bursts Huntsville. W.S. Paciesas \& G.J. Fishman Eds., p.22

Yoshida, S., et al. 1995, Astropart. Phys., 3105 